\title{
Socioeconomic status and survival in patients with pulmonary hypertension
}

\author{
Mei-Sing Ong (i) \\ Dept of Population Medicine, Harvard Medical School \& Harvard Pilgrim Health Care Institute, Boston MA, \\ USA.
}

Correspondence: Mei-Sing Ong, Dept of Population Medicine, Harvard Medical School and Harvard Pilgrim Health Care Institute, 401 Park Drive, Suite 401E, Boston, MA 02115, USA.

E-mail: Mei-Sing_Ongahms.harvard.edu

@ERSpublications

In a publicly funded healthcare system, no evidence of survival disparities across socioeconomic classes among patients with pulmonary hypertension was observed, underscoring the importance of eliminating financial barriers to medical care and treatment https://bit.ly/2Eb1ju2

Cite this article as: Ong M-S. Socioeconomic status and survival in patients with pulmonary hypertension. ERJ Open Res 2020; 6: 00638-2020 [https://doi.org/10.1183/23120541.00638-2020].

Socioeconomic status is one of the most crucial determinants of health status in virtually every society. Even in high-income countries, disparities in health across socioeconomic backgrounds are pronounced. A study conducted by the World Health Organization reported an association of low socioeconomic status with a life expectancy reduction of $>2$ years, greater than that for high alcohol intake, obesity and hypertension [1]. Recent studies have also highlighted socioeconomic status as a significant predictor of survival in patients with pulmonary hypertension [2-4]. Given the considerable financial burden associated with the treatment of pulmonary hypertension $[5,6]$, these findings are hardly surprising.

In this issue of ERJ Open Research, MCGETTRICK et al. [7] evaluated the relationship between social deprivation and survival outcome in patients with connective tissue disease-associated pulmonary hypertension (CTD-PH) and chronic thromboembolic pulmonary hypertension (CTEPH). The study defined social deprivation in accordance with the Scottish Index of Multiple Deprivation (SIMD), an area-based socioeconomic indicator developed by the Scottish Government based on seven domains: income, employment, health, education, skills and training, geographical access to services, crime, and housing. Reviewing the charts of patients with CTD-PH $(n=232)$ and CTEPH $(n=263)$ under the care of the Scottish Pulmonary Vascular Unit (SPVU) between 1992 and 2018, the authors found that increased social deprivation was not associated with poorer survival in these patients. The study further found no differences in the presenting functional class of patients with CTD-PH, suggesting that there was no evidence of inequities in treatment access across socioeconomic classes. CTEPH patients living in socially deprived neighbourhood were, however, more likely to present with a worse functional class at the time of diagnosis. The authors concluded that social deprivation was not a significant prognostic factor for CTD-PH and CTEPH in Scotland. The results of this study are consistent with another study, also conducted at the SPVU, reporting the absence of socioeconomic disparities in the survival outcome of patients with idiopathic pulmonary arterial hypertension [8].

These findings stand in stark contrast to a growing body of evidence demonstrating substantial socioeconomic gradient in life expectancy both in the general population $[1,9]$ and across a spectrum of chronic diseases, including pulmonary hypertension. Published studies in the USA reported poorer survival among pulmonary hypertension patients with a lower household income [2] and those residing in rural areas [3]. A study in China also reported higher mortality rate among socioeconomically disadvantaged patients with pulmonary hypertension [4]. The authors attributed the contrasting study outcomes to the differences in healthcare system: while Scotland has a publicly funded healthcare system whereby access to

Received: 3 Sept 2020 | Accepted: 13 Sept 2020

Copyright $\odot$ ERS 2020. This article is open access and distributed under the terms of the Creative Commons Attribution Non-Commercial Licence 4.0. 
healthcare and medical treatment are free at the point of delivery, the USA and China do not have universal health insurance coverage. In the USA, a substantial number of residents remain uninsured; even among those who are insured, insurance coverage of medical services and treatments varies widely, and are often accompanied by high out-of-pocket costs. The high cost of pulmonary hypertension therapy can be a barrier to treatment access for the uninsured. Although many factors confound cross-national comparisons, published studies in the USA and other countries have consistently shown that uninsured individuals have poorer health and life expectancy than insured individuals, and expansion of health insurance coverage reduces mortality burden in the most vulnerable populations [10-14].

Financial barriers to medical treatment are, however, only one of many drivers of health inequities. Disparities in health among socioeconomic classes have also been reported in healthcare systems where access to care is presumably universal $[15,16]$. How much of the better health of the privileged is accounted for by better access to healthcare remains difficult to quantify. In Scotland, published studies reported disparities in health outcomes across a range of conditions, including cancer [17], cardiovascular disease $[18,19]$ and type 1 diabetes [20]. What differentiates the current study may be that treatment of pulmonary hypertension in Scotland is only available at one centre to which all patients in the country with pulmonary hypertension are referred. As highlighted by PeLlino et al. [8], this centralisation of care enhances the likelihood of care uniformity regardless of a patient's postcode. In most other settings, socioeconomically disadvantaged individuals are more likely to receive care at medical centres with fewer resources available to provide high-quality care, and greater access to high-quality hospitals offsets socioeconomic disparities in survival for a range of conditions [21].

Any observational study is susceptible to confounding by indication and the results of the current study need to be interpreted in the light of several limitations. First, referral bias may have led to fewer socioeconomically disadvantaged patients being seen at the SPVU. The authors attempted to address this potential bias by comparing the distribution of social deprivation status among the study cohort against the general population in Scotland. The comparison showed no differences, which is reassuring. Second, the current study uses the SIMD, an area-based social deprivation indicator, as a proxy for individual socioeconomic status. Although area-based measures can meaningfully capture socioeconomic context at the neighbourhood scale, it cannot identify whether individuals or households are experiencing deprivation. A study evaluating the SIMD showed that the proportion of deprived individuals within areas designated as deprived varies widely across different regions [22]. When applied to a relatively small study cohort, this limitation may not be negligible [23]. Third, the effects of socioeconomic status on health outcomes can vary substantially by population groups not accounted for in the current study. Most notably, racial and ethnic minorities often experience undesirable health outcomes. Although members of minority populations are disproportionately more likely to have low socioeconomic status, published studies have shown that race and ethnicity are independent predictors of poor health outcomes. For example, a USA-based study found that pregnancy-related mortality rate for black women with at least a college degree was 1.6 times that of white women with less than a high school diploma and five times that of white college-educated women [24]. Racial disparities in health outcomes have also been documented in Scotland [25-27]. The association of race/ethnicity and survival in pulmonary hypertension is less well understood. Most evidence to date points to a trend towards increased mortality among minority races compared with white people [28-30]; however, data from the REVEAL registry demonstrated a higher mortality risk among white people compared with minority races [31]. Racial and ethnic disparities are arguably the most obstinate inequities in health over time, driven by long-standing systemic social inequities. A closer look at the interactions between socioeconomic status and race/ethnicity will allow for more robust explanations of the patterns of disparities.

The pathways through which socioeconomic status affect healthcare are complex. Nonetheless, the consequences of socioeconomic status on health are modifiable by policies. Although more research is needed to understand the influence of socioeconomic status on survival in pulmonary hypertension, the study by McGeTTRICK et al. [7] offers an encouraging example of how providing equal access to healthcare can potentially help address survival disparities in a high-morbidity disease. The current COVID-19 pandemic has laid to bare the widespread societal consequences of health inequities. In this time of worsening socioeconomic conditions and rising inequality, widening disparities could result. The importance of addressing health inequities and disparities cannot be overstated.

Conflict of interest: M-S. Ong has nothing to disclose.

\section{References}

1 Stringhini S, Carmeli C, Jokela M, et al. Socioeconomic status and the $25 \times 25$ risk factors as determinants of premature mortality: a multicohort study and meta-analysis of 1.7 million men and women. Lancet 2017 ; 389: $1229-1237$. 
2 Talwar A, Sahni S, Talwar A, et al. Socioeconomic status affects pulmonary hypertension disease severity at time of first evaluation. Pulm Circ 2016; 6: 191-195.

3 Macías CG, Wharam JF, Maron BA, et al. Urban-Rural Disparities in Pulmonary Hypertension Mortality. Ann Am Thorac Soc 2020; 17: 1168-1171.

4 Wu W-H, Yang L, Peng F-H, et al. Lower socioeconomic status is associated with worse outcomes in pulmonary arterial hypertension. Am J Respir Crit Care Med 2013; 187: 303-310.

5 Sikirica M, Iorga SR, Bancroft T, et al. The economic burden of pulmonary arterial hypertension (PAH) in the US on payers and patients. BMC Health Serv Res 2014; 14: 676.

6 Burke JP, Hunsche E, Régulier E, et al. Characterizing pulmonary hypertension-related hospitalization costs among Medicare Advantage or commercially insured patients with pulmonary arterial hypertension: a retrospective database study. Am J Manag Care 2015; 21: s47-s58.

7 McGettrick M, McCaughey P, MacLellan A, et al. Social deprivation in Scottish populations with pulmonary hypertension secondary to connective tissue disease and chronic thromboembolic disease. ERJ Open Research 2020; 6: 00297-2019.

8 Pellino K, Kerridge S, Church C, et al. Social deprivation and prognosis in Scottish patients with pulmonary arterial hypertension. Eur Respir J 2018; 51: 1700444.

9 Woolf SH, Schoomaker H. Life expectancy and mortality rates in the United States, 1959-2017. JAMA 2019; 322: 1996-2016.

10 Woolhandler S, Himmelstein DU. The relationship of health insurance and mortality: is lack of insurance deadly? Ann Intern Med 2017; 167: 424-431.

11 Miller S, Altekruse S, Johnson N, et al. Medicaid and Mortality: New Evidence from Linked Survey and Administrative Data. Cambridge, National Bureau of Economic Research, 2019.

12 Bhatt CB, Beck-Sagué CM. Medicaid expansion and infant mortality in the united states. Am J Public Health 2018; 108: 565-567.

13 Wen CP, Tsai SP, Chung W-SI. A 10-year experience with universal health insurance in Taiwan: measuring changes in health and health disparity. Ann Intern Med 2008; 148: 258-267.

14 Hanratty MJ. Canadian national health insurance and infant health. Am Econ Rev 1996; 86: 276-284.

15 Blendon RJ, Schoen C, DesRoches CM, et al. Inequities in health care: a five-country survey. Health Aff (Millwood) 2002; 21: 182-191.

16 Katz SJ, Hofer TP. Socioeconomic disparities in preventive care persist despite universal coverage. Breast and cervical cancer screening in Ontario and the United States. JAMA 1994; 272: 530-534.

17 Tweed EJ, Allardice GM, McLoone P, et al. Socio-economic inequalities in the incidence of four common cancers: a population-based registry study. Public Health 2018; 154: 1-10.

18 O'Flaherty M, Bishop J, Redpath A, et al. Coronary heart disease mortality among young adults in Scotland in relation to social inequalities: time trend study. BMJ 2009; 339: b2613.

19 Hotchkiss JW, Davies C, Gray L, et al. Trends in adult cardiovascular disease risk factors and their socio-economic patterning in the Scottish population 1995-2008: cross-sectional surveys. BMJ Open 2011; 1: e000176.

20 Campbell RAS, Colhoun HM, Kennon B, et al. Socio-economic status and mortality in people with type 1 diabetes in Scotland 2006-2015: a retrospective cohort study. Diabet Med 2020; in press [https://doi.org/10.1111/ dme.14239].

21 Kahn KL, Pearson ML, Harrison ER, et al. Health care for black and poor hospitalized Medicare patients. JAMA 1994; 271: 1169-1174.

22 Clelland D, Hill C. Deprivation, policy and rurality: The limitations and applications of area-based deprivation indices in Scotland. Local Economy 2019; 34: 33-50.

23 Greenwald HP, Polissar NL, Borgatta EF, et al. Detecting survival effects of socioeconomic status: problems in the use of aggregate measures. J Clin Epidemiol 1994; 47: 903-909.

24 Petersen EE, Davis NL, Goodman D, et al. Racial/ethnic disparities in pregnancy-related deaths - United States, 2007-2016. MMWR Morb Mortal Wkly Rep 2019; 68: 762-765.

25 Katikireddi SV, Cezard G, Bhopal RS, et al. Assessment of health care, hospital admissions, and mortality by ethnicity: population-based cohort study of health-system performance in Scotland. Lancet Public Health 2018; 3: e226-e236.

26 Sheikh A, Steiner MFC, Cezard G, et al. Ethnic variations in asthma hospital admission, readmission and death: a retrospective, national cohort study of 4.62 million people in Scotland. BMC Med 2016; 14: 3 .

27 Bhopal RS, Bansal N, Steiner M, et al. Does the "Scottish effect" apply to all ethnic groups? All-cancer, lung, colorectal, breast and prostate cancer in the Scottish Health and Ethnicity Linkage Cohort Study. BMJ Open 2012; 2: e001957.

28 Kawut SM, Horn EM, Berekashvili KK, et al. New predictors of outcome in idiopathic pulmonary arterial hypertension. Am J Cardiol 2005; 95: 199-203.

29 George MG, Schieb LJ, Ayala C, et al. Pulmonary hypertension surveillance: United States, 2001 to 2010. Chest 2014; 146: 476-495.

30 Ong M-S, Abman S, Austin ED, et al. Racial and ethnic differences in pediatric pulmonary hypertension: an analysis of the pediatric pulmonary hypertension network registry. J Pediatr 2019; 211: 63-71.e6.

31 Farber HW, Miller DP, Poms AD, et al. Five-year outcomes of patients enrolled in the REVEAL Registry. Chest 2015; 148: 1043-1054. 